\title{
Toll like receptor 4 plays significant roles during allergic rhinitis
}

Masoud Radman ${ }^{1}$, Ali Golshiri Isfahani ${ }^{1}$, Ali Shamsizadeh², Nahid Zainodini $^{3}$, Mohammad Kazemi Arababadi ${ }^{3 *}$

1. Dept of Otolaryngology, Faculty of Medicine, Rafsanjan University of Medical Sciences, Rafsanjan, Iran.

2. Physiology-Pharmacology Research Center, Rafsanjan University of Medical Sciences, Rafsanjan, Iran.

3. Immunology of Infectious Diseases Research Center, Rafsanjan University of Medical Sciences, Rafsanjan, Iran.

*Corresponding author:

Dr. Mohammad Kazemi Arababadi, Immunology of Infectious Diseases Research Center, Rafsanjan University of Medical Sciences, Rafsanjan, Iran. E-mail: dr.kazemi@rums.ac.ir

Tel: 00983915234003-5

Mobile: 00989132926113

Fax: 00983915225209

Running title: TLR4 and allergic rhinitis 


\begin{abstract}
Allergic rhinitis is a nasal hypersensitivity and allergic diseases which leads to inflammation of nasal mucosa. Previous investigations revealed that innate immune receptors play a key role in the pathogenesis of inflammatory diseases including allergic diseases. Toll like receptors (TLRs), which are important innate immune receptors, play crucial roles in recognition of foreign antigens, including allergens, and subsequently for the induction of immune responses such as inflammation. There are several controversial reports regarding the roles of TLR4 in the pathogenesis of allergic rhinitis. This review presents current information regarding the roles of TLR4 in the pathogenesis of allergic rhinitis and the plausible mechanisms which lead to the expression and function of TLR4 in this disease.
\end{abstract}

Key words: Allergic rhinitis, TLR4, Inflammation.

\title{
Abbreviation:
}

AP-1: activator protein 1. DAMP: damage associated molecular patterns. IRAK1: Interleukin-1 receptor associated kinase-1. LBP: LPS binding protein. LPS: Lipopolysacharide. MAPK: Mitogen-activated protein kinase. MD2: Myeloid differentiation factor 2. MHC: Major histocompatibility complex. MYD88: Myeloid differentiation primary response. NADPH: Nicotinamide adenine dinucleotide phosphate. NF-kB: Nuclear factor kappa-light-chain-enhancer of activated B cells. PAMP: pathogen associated molecular patterns. PRR: pathogen recognition receptors. TAK1: Transforming growth factor b-activated kinase 1. TIRAP: TIR domain-containing adaptor protein. TLR: Toll like receptor. TRAF6: TNF receptor associated factor. TRAM: TRIF-related adaptor molecule. TRIF: TIR-domain-containing adapter-inducing interferon- $\beta$.

\section{Introduction}


Toll-Like Receptors (TLRs) are the main intra/extra-cellular immune cell receptors which belong to Pathogen Recognition Receptor (PRR) family. These molecules play crucial roles in recognition of pathogen associated molecular patterns (PAMPs) and damage associated molecular patterns (DAMPs) and subsequently facilitate the induction of immune responses such as inflammation (1).

TLR/PAMP or DAMP interactions result in various alterations in immune cell function and surface molecules including expression of homing molecules (2), NADPH oxidase activation (3) and inflammatory cytokine secretion (4). Like other TLRs, TLR4 acts via interaction with its corresponding ligands (see next sections) in both MYD88 and TRIF dependent manners (5).

Allergic rhinitis is an allergic disease of nasal airways which is associated with inflammatory conditions and it may be hypothesized that TLR4 may play critical roles in the pathogenesis of allergic diseases such as allergic rhinitis. Therefore, the aim of this study was to review recent information regarding the relationship between TLR4 and allergic rhinitis. This study also presents recent information regarding the possible mechanisms leading to alterations in expression of TLR4 and its signaling molecules in these patients. 


\section{Allergic rhinitis; an inflammatory disease}

Allergic rhinitis, which is also known as nasal allergy and nasal hypersensitivity, is defined as an allergic condition of the nasal airways (6,

7). It can be induced in individuals with a sensitized immune system via inhalation of an allergen, including pollen and dust or particles of shed skin and hair of animals (animal dander) (8). In sensitive individuals, the allergen stimulates the production of IgE which binds to FCeRI on mast cells and basophils and induces secretion of several mediators including histamine, leukotrienes, prostaglandins and associated enzymes including tryptase (8). These mediators cause nasal passages swelling, paroxysmal repetitive sneezing, itchy and watery eyes and elevation in mucus production, via stimulation of the mucous glands and vascular permeability (known as primary phase) and activation and perpetuation of inflammatory cells (late phase) (9). The severity of symptoms is varying between individuals. Pollinosis and hay fever are two forms of allergic rhinitis caused by pollens of any plants and grass pollens, respectively $(10,11)$. It has been documented that genetic, immunological and environmental parameters play important roles in the pathogenesis of allergic rhinitis (12). Allergic rhinitis is prevalent worldwide and investigators estimate that 30 percent of people experience this disease at least once in their lives (13). Depending on the 
causative antigens, period of exposure, severity of symptoms and disease types, allergic rhinitis is classified to several clinical manifestations. The most commonly used classification use perennial and seasonal occurrence to define the disorder (14). There are various forms of therapies for allergic rhinitis as follow: 1. Oral antihistamines, 2. Topical antihistamines, 3. Decongestants (via activating alpha-adrenergic receptors), 4. Intranasal glucocorticoids, 5. Leukotriene receptor antagonists and 6. Intranasal cromolyns (by stabilization and inhibition the degranulation of mast cells) $(15,16)$. The current therapies are associated with several side effects and in some cases patients present drug resistance, hence, novel therapies must be explored for the treatment of this disease (17). In order to design a successful therapy, it is important to understand the roles played by immunerelated molecules.

\section{TLR4; structure, genomic location and agonists}

TLR4, which is also known as CD284 or ARMD10, is the receptor for several microbial PAMPs, which will be discussed below. The TLR4 protein consists of three complex domains including extracellular leucine-rich repeats (LRRs), a hydrophobic transmembrane and an intracytoplasmic Toll/interleukin-1 receptor (TIR) domain. TLR4 recognizes several 
microbial and non-microbial molecules as a homodimer which leads to activation of various intracellular signaling pathways (See next sections).

Various molecules have been identified as agonists for TLR4 (19). LPS is the most important agonist of TLR4 and is recognized by a TLR4/CD14/MD2 complex (20). LPS binding protein (LBP) is responsible for binding LPS to a Glycosylphosphatidylinositol-anchored protein called CD14 which subsequently delivers the LPS to a soluble protein, MD2 (also known as lymphocyte antigen 96) (20). MD2 binds non-covalently to the extracellular domain of TLR4 inducing a conformational change in the MD2 structure which results in binding of the MD-2-TLR4 complex to a second TLR4 molecule (20). Following TLR4 homo-dimerization, TRIF and MYD88 dependent intracellular signaling pathways will be activated (see next section). Short ragweed (SRW) pollen, high-mobility group box-1, hyaluronan, heat shock protein 60 , free fatty acids, allergenic nickel and the adjuvant monophosphoryl lipid A (MPLA) are agonists for TLR4 which are used as adjuvants for the induction of immune responses against vaccination (21-23). Interestingly, high-mobility group box-1, hyaluronan, heat shock protein 60 and free fatty acids, which are endogenous TLR4 ligands, not only activate TLR4 directly, but can also bind and transport LPS to TLR4 
which increases cell sensitivity to LPS suggesting that endogenous TLR4 ligands can be considered as PAMP binding/sensitizing molecules (24).

\section{TLR4 signaling}

Interactions of TLR4 and its corresponding ligands leads to the recruitment of TIRAP/MYD88 and TRAM/TRIF, TIR-containing adaptor molecules, which participate in stimulation MYD88 and TRIF-dependent signaling pathways, respectively (25). Previous in vitro and in vivo studies demonstrated that TLR4/ligands interaction at the plasma membrane leads to recruitment of TIRAP which allows binding of the adaptor protein, MyD88. Following recruitment of MYD88 several intracellular signaling molecules such as IRAK4, IRAK1, TRAF6, TAK1, NF-kB, AP-1 and IRF5 are activated (19). NF-kB, AP-1 and IRF5 are transcription factors that induce transcription of several pro-inflammatory cytokines (26). Additionally, upon internalization of TLR4 to the endosomes and interactions with its agonists, another signaling pathway, the TRIF-dependent pathway, is activated leading to binding of the TRAM TIR domain to the TIR domain of TLR4 (26). TRAF3 and IRP1 molecules are activated (phosphorylated) following TRIF binding to TRAM (27). Phosphorylation of TRAF3 results in activation of another transcription factor, IRF3, and leads to translocation of this molecule into the nucleus to induce transcription of type I interferons 
(26). IRP1 is also activated in the TRIF dependent pathway which leads to activation of other transcription factors such as AP-1 and NF- $\mathrm{BB}$ (27).

\section{TLR4 and allergic rhinitis:}

The activation of several pro-inflammatory transcription factors and the stimulation of pro-inflammatory cytokine production through TLR4 signaling suggests that it can participate in several inflammatory conditions such as allergic diseases. However, its role in the pathogenesis of allergic rhinitis is controversial. Some studies demonstrated that expression levels of TLR4 increased in allergic rhinitis. For instance, Fransson et al., have studied the relationship between the expression of TLR4 in allergic rhinitis and reported a significant upregulation of TLR4, at both the mRNA and protein levels, in the nasal mucosa of the patients (28). Lin and Ming demonstrated that expression levels of TLR4 and its corresponding transcription factor, NF- $\kappa \mathrm{B}$, increased in rat nasal mucosa in an experimental allergic rhinitis model (29). Another study reported that up-regulation of TLR4 can lead to lipopolysaccharide-induced augmentation of nasal cytokine release in allergic rhinitis (30). Li and co-workers showed that Short Ragweed (SRW) pollen can induce allergic rhinitis via activation of TSLP/OX40L/OX40 signaling and increased production of Th2 cytokine in a TLR4-dependent manner (23). These results suggest that TLR4 may 
participate significantly in the pathogenesis of allergic rhinitis. However, it seems that this is not all of story and negative as well as non-association of TLR4 with allergic rhinitis have been reported repeatedly. For example, in contrast to the aforementioned studies, Lauriello et al., reported that TLR4 expression was decreased significantly in allergic rhinitis patients (31). Vanhinsbergh and colleagues have also revealed that TLR4 was downregulated during allergic rhinitis (32). Interestingly, Ryu et al., also showed that LPS is not essential to activate innate immunity in the nasal mucosa of patients with allergic rhinitis (33) and it may be related to down-regulation of TLR4 in these patients. It has also been documented that mRNA levels of TLR4 decreased in allergic rhinitis (32). In addition to the data reviewed here, several investigations demonstrated the controversial roles of genetic variations within the TLR4 gene in the pathogenesis of allergic rhinitis. For instance, Fuertes et al., revealed that the rs1927911 polymorphism within the TLR4 gene is associated with higher risk of allergic rhinitis (34). Although, Hussein et al., reported that there is not an association between TLR4 polymorphisms and allergic rhinitis but they have suggested that the polymorphism can be significantly associated with disease severity (35). Negative associations of TLR4 with allergic rhinitis were also reported by Kurowski et al., who demonstrated that CD14/-159CC and CD14/-159TT 
genotypes were associated with reduced incidence of allergic rhinitis in children (36). Another investigation reported that the frequencies of 4216G >C genotypes and alleles in the TLR4 gene were not associated with allergic rhinitis in a Chinese population (37). Another study on Chinese populations demonstrated that Asp299Gly and Thr399Ile polymorphisms within the TLR4 gene were not detected in patients with allergic rhinitis (38). Fagerås Böttcher and colleagues also showed that the TLR4 (Asp299Gly) polymorphism was not associated with allergic rhinoconjunctivitis (39). Eder et al., reported that the polymorphisms at +4434 position of TLR4 gene were not associated with allergic rhinitis (40). Due to the results presented here, it appears that participation of TLR4 polymorphisms in the pathogenesis of allergic rhinitis is controversial. It may be hypothesized that various races and ethnic backgrounds in the evaluated population of these studies and the techniques which have been used to determine the polymorphisms may be reasons for the discrepancies between these studies. Accordingly, it may be hypothesized that the role of TLR4 in the induction of inflammation during allergic rhinitis is dependent on the following mechanisms: Based on the aforementioned data, SRW pollen is capable of inducing allergic rhinitis via activation of TSLP/OX40L/OX40 signaling and increased production of Th2 cytokine in a TLR4-dependent manner (23), 
hence, it may be concluded that the various allergens can induce inflammation via different immune cell receptors and their corresponding intracellular signaling. Thus, it may be hypothesized that some of allergens can be recognized by TLR4 and induce inflammation in allergic rhinitis, therefore, TLR4 may play important roles in the pathogenesis of allergic rhinitis induced by some specific allergens such as SRW pollen. Moreover, positive or negative roles of TLR4 in the pathogenesis of allergic rhinitis may be related to the severity of the disease which has previously been reported (35). Based on the studies on the genetic variations of TLR4, it seems that some of polymorphisms may be associated with allergic rhinitis, thus, genetic variation may be considered as another mechanism responsible for the induction of allergic rhinitis in some patients. Accordingly, the authors of the current review article suggests that reduction in TLR4 gene expression in the patients with allergic rhinitis, which is induced by specific allergens, such as SRW pollen, may protect patients against the development of allergic rhinitis. Recently, several disorders have been treated by using monoclonal antibodies against target molecules $(41,42)$. It is intriguing to consider the possibility of using topically applied antibodies to regulate TL4 signaling to regulate the pathogenesis of allergic rhinitis. Furthermore, it has been established that the TLR4 agonist, CRX-657 induces Th1 cell 
responses, hence, it may be used to create a reciprocal reduction in Th2 responses during allergic diseases. It has been reported that treatment with CRX-657 decreased nasal congestion after allergen challenge in an animal model of allergic rhinitis. Casale, et al., demonstrated that there was no severe effect after intranasal application of CRX-657. However, more clinical investigations are needed to improve its potential role as a therapeutic agent against allergic disorders including allergic rhinitis. These data would suggest that all molecules which alter TLR4 expression could be associated with the pathogenesis of this disease. For instance, Bondeva et al., revealed that angiotensin II (ANG II) increased expression of TLR4 via interactions with AP1 and PU.1 sites (느). Furthermore, ANG II leads to contraction of human airway smooth muscle cells (4ㅗ) which has also been observed during several allergic disorders including asthma (ㄴ5). There are apparently several possible mechanisms involved in the disease progression, and ANG II represents only one plausible pathway leading to the upregulation of TLR4 with implications for the development of allergic rhinitis. More investigations are needed to improve our knowledge regarding the molecular mechanisms of allergic rhinitis.

\section{Conclusions}


TLR4 shows different patterns of expression and is complicated by it various implicated roles in allergic rhinitis, which change depending on the nature of allergen, severity of the disease and genetic variation of the patients. One pathway towards development of a new therapeutic for the disease could focus around TLR4's pivotal role in controlling allergic reactions and studies directed towards the use of agonists or antagonists of TLR4 during the etiology of allergic rhinitis could provide the clues needed for treatment of the disease.

\section{Acknowledgments:}

This project was supported by the Research committee of Rafsanjan University of Medical Sciences (RUMS).

\section{Declaration of Interest: None}

\section{References}

1. Khvalevsky E, Rivkin L, Rachmilewitz J, Galun E, Giladi H. TLR3 signaling in a hepatoma cell line is skewed towards apoptosis. J Cell Biochem. 2007;100(5):1301-12. Epub 2007/01/24.

2. $\quad$ Nguyen-Pham TN, Lim MS, Nguyen TA, Lee YK, Jin CJ, Lee HJ, et al. Type I and II interferons enhance dendritic cell maturation and migration capacity by regulating CD38 and CD74 that have synergistic effects with TLR agonists. Cell Mol Immunol. 2011;8(4):341-7.

3. Bae YS, Lee JH, Choi SH, Kim S, Almazan F, Witztum JL, et al. Macrophages generate reactive oxygen species in response to minimally oxidized low-density lipoprotein: toll-like receptor 4- and spleen tyrosine kinase-dependent activation of NADPH oxidase 2. Circ Res. 2009;104(2):210-8, 21p following 8.

4. Imani Fooladi AA, Mousavi SF, Seghatoleslami S, Yazdani S, Nourani MR. Tolllike receptors: role of inflammation and commensal bacteria. Inflamm Allergy Drug Targets. 2011;10(3):198-207. 
5. Jia N, Xie Q, Lin L, Gui H, Wang H, Jiang S, et al. Common variants of the TLR9 gene influence the clinical course of HBV infection. Mol Med Report. 2009;2(2):277-81.

6. Whittaker C. Allergic rhinitis: review. 2010.

7. Greiner AN, Hellings PW, Rotiroti G, Scadding GK. Allergic rhinitis. The Lancet. 2012;378(9809):2112-22.

8. $\quad$ Shiung Y-Y, Chiang C-Y, Chen J-B, Wu PC, Hung AF-H, Lu DC-S, et al. An anti-IgE monoclonal antibody that binds to IgE on CD23 but not on high-affinity IgE. Fc receptors. Immunobiology. 2012;217(7):676-83.

9. Songu M. Physiology and Pathophysiology of Sneezing and Itching: Mechanisms of the Symptoms. Nasal Physiology and Pathophysiology of Nasal Disorders: Springer; 2013. p. 139-52.

10. Croft AM, Bager P, Kumar S. Helminth therapy (worms) for allergic rhinitis. Cochrane Database Syst Rev. 2012;4.

11. Wüthrich B, Schmid-Grendelmeier P, Schindler C, Imboden M, Bircher A, Zemp $\mathrm{E}$, et al. Prevalence of atopy and respiratory allergic diseases in the elderly SAPALDIA population. International archives of allergy and immunology. 2013;162(2):143-8.

12. Boyce JA, Bochner B, Finkelman FD, Rothenberg ME. Advances in mechanisms of asthma, allergy, and immunology in 2011. Journal of Allergy and Clinical Immunology. 2012;129(2):335-41.

13. Żukiewicz-Sobczak W, Krasowska E, Zwoliński J, Sobczak P, ChmielewskaBadora J, Wróblewska P, et al. Review paper Allergic diseases-current state of knowledge. 2012.

14. Scheerens H, Harris JM, Matthews J, Putnam WS, Liao XC. Anti-ige antibodies and methods using same. US Patent App. 13/754,572; 2013.

15. Lim M, Leong J. Allergic rhinitis: evidence-based practice. Singapore Med J. 2010;51(7):542-50.

16. Corriveau M-N, Bachert C. 6 Allergic and Nonallergic Rhinitis. Rhinology: Diseases of the Nose, Sinuses, and Skull Base. 2012.

17. SAWADA T, ARIMURA A, KUWAJIMA G. DRUG FOR THE TREATMENT OF ALLERGIC RHINITIS COMPRISING PGD2 ANTAGONIST AND HISTAMINE ANTAGONIST. WO Patent 2,013,047,725; 2013.

18. Qureshi ST, Larivière L, Leveque G, Clermont S, Moore KJ, Gros P, et al. Endotoxin-tolerant mice have mutations in Toll-like receptor 4 (Tlr4). The Journal of experimental medicine. 1999;189(4):615-25.

19. Yamamoto M, Sato S, Hemmi H, Hoshino K, Kaisho T, Sanjo H, et al. Role of adaptor TRIF in the MyD88-independent toll-like receptor signaling pathway. Science. 2003;301(5633):640-3.

20. Kagan JC, Medzhitov R. Phosphoinositide-mediated adaptor recruitment controls Toll-like receptor signaling. Cell. 2006;125(5):943-55.

21. Evans JT, Cluff CW, Johnson DA, Lacy MJ, Persing DH, Baldridge JR. Enhancement of antigen-specific immunity via the TLR4 ligands MPL ${ }^{\mathrm{TM}}$ adjuvant and Ribi. 529. Expert review of vaccines. 2003;2(2):219-29.

22. Alderson MR, McGowan P, Baldridge JR, Probst P. TLR4 agonists as immunomodulatory agents. Journal of Endotoxin Research. 2006;12(5):313-9. 
23. Li D-Q, Zhang L, Pflugfelder SC, De Paiva CS, Zhang X, Zhao G, et al. Short ragweed pollen triggers allergic inflammation through Toll-like receptor 4-dependent thymic stromal lymphopoietin/OX40 ligand/OX40 signaling pathways. Journal of Allergy and Clinical Immunology. 2011;128(6):1318-25. e2.

24. Erridge C. Endogenous ligands of TLR2 and TLR4: agonists or assistants? J Leukoc Biol. 2010;87(6):989-99. Epub 2010/02/25.

25. Cario E, Gerken G, Podolsky D. Toll-like receptor 2 controls mucosal inflammation by regulating epithelial barrier function. Gastroenterology. 2007;132(4):1359-74.

26. Fitzgerald KA, Rowe DC, Barnes BJ, Caffrey DR, Visintin A, Latz E, et al. LPSTLR4 signaling to IRF-3/7 and NF- $\mathrm{B}$ involves the Toll adapters TRAM and TRIF. The Journal of experimental medicine. 2003;198(7):1043-55.

27. Lu Y-C, Yeh W-C, Ohashi PS. LPS/TLR4 signal transduction pathway. Cytokine. 2008;42(2):145-51.

28. Fransson M, Adner M, Erjefält J, Jansson L, Uddman R, Cardell L-O. Upregulation of Toll-like receptors 2, 3 and 4 in allergic rhinitis. Respiratory research. 2005;6(1):100.

29. Lin Z, Ming Z. Expression of Toll-Like Receptor 4 and NF- $\mathrm{Bp50}$ in Experimental Allergic Rhinitis in Rats. Sichuan Journal of Anatomy. 2010;1:002.

30. Ekman AK, Virtala R, Fransson M, Adner M, Benson M, Jansson L, et al. Systemic up-regulation of TLR4 causes lipopolysaccharide-induced augmentation of nasal cytokine release in allergic rhinitis. Int Arch Allergy Immunol. 2012;159(1):6-14. Epub 2012/05/05.

31. Lauriello M, Micera A, Muzi P, Di Rienzo Businco L, Bonini S. TLR4 and TLR9 Expression in Different Phenotypes of Rhinitis. International Journal of Otolaryngology. 2012;2012.

32. Vanhinsbergh LJ, Powe DG, Jones NS. Reduction of TLR2 gene expression in allergic and nonallergic rhinitis. Annals of Allergy, Asthma \& Immunology. 2007;99(6):509-16.

33. Ryu JH, Yoo JY, Kim MJ, Hwang SG, Ahn KC, Ryu JC, et al. Distinct TLRmediated pathways regulate house dust mite-induced allergic disease in the upper and lower airways. J Allergy Clin Immunol. 2013;131(2):549-61. Epub 2012/10/06.

34. Fuertes E, Brauer M, MacIntyre E, Bauer M, Bellander T, von Berg A, et al. Childhood allergic rhinitis, traffic-related air pollution, and variability in the GSTP1, TNF, TLR2, and TLR4 genes: results from the TAG Study. J Allergy Clin Immunol. 2013;132(2):342-52 e2. Epub 2013/05/04.

35. Hussein YM, Awad HA, Shalaby SM, Ali AS, Alzahrani SS. Toll-like receptor 2 and Toll-like receptor 4 polymorphisms and susceptibility to asthma and allergic rhinitis: a case-control analysis. Cell Immunol. 2012;274(1-2):34-8. Epub 2012/03/10.

36. Kurowski M, Majkowska-Wojciechowska B, Wardzyńska A, Kowalski ML. Associations of allergic sensitization and clinical phenotypes with innate immune response genes polymorphisms are modified by house dust mite allergen exposure. Arch Med Sci. 2011;7:1029-36.

37. Kang I, Oh Y-K, Lee SH, Jung HM, Chae S-C, Lee JH. Identification of polymorphisms in the Toll-like receptor gene and the association with allergic rhinitis. European Archives of Oto-Rhino-Laryngology. 2010;267(3):385-9. 
38. Liang XH, Cheung W, Heng CK, Wang DY. Absence of the toll-like receptor 4 gene polymorphisms Asp299Gly and Thr399Ile in Singaporean Chinese. Ther Clin Risk Manag. 2005;1(3):243-6. Epub 2008/03/25.

39. Fagerås Böttcher M, Hmani-Aifa M, Lindström A, Jenmalm MC, Mai X-M, Nilsson $\mathrm{L}$, et al. A<i $>$ TLR4 $</ \mathrm{i}>$ polymorphism is associated with asthma and reduced lipopolysaccharide-induced interleukin-12 (p70) responses in Swedish children. Journal of allergy and clinical immunology. 2004;114(3):561-7.

40. Eder W, Klimecki W, Yu L, von Mutius E, Riedler J, Braun-Fahrländer C, et al. Toll-like receptor 2 as a major gene for asthma in children of European farmers. Journal of Allergy and Clinical Immunology. 2004;113(3):482-8.

41. Zaza G, Tomei P, Granata S, Boschiero L, Lupo A. Monoclonal antibody therapy and renal transplantation: focus on adverse effects. Toxins (Basel). 2014;6(3):869-91. Epub 2014/03/05.

42. Wattenberg MM, Kwilas AR, Gameiro SR, Dicker AP, Hodge JW. Expanding the use of monoclonal antibody therapy of cancer by using ionising radiation to upregulate antibody targets. Br J Cancer. 2014. Epub 2014/02/22.

43. Bondeva T, Roger T, Wolf G. Differential regulation of Toll-like receptor 4 gene expression in renal cells by angiotensin II: dependency on AP1 and PU. 1 transcriptional sites. American journal of nephrology. 2007;27(3):308-14.

44. Li N, Meng Y, Cheng Y, Niu Y, Shen B, Li X, et al. [Activation of Rho-kinase pathway is involved in angiotensin II-induced contraction of human airway smooth muscle cells]. Nan fang yi ke da xue xue bao= Journal of Southern Medical University. 2011;31(8):1416-20.

45. Abousaidi H, Vazirinejad R, Arababadi MK, Rafatpanah H, Pourfathollah AA, Derakhshan R, et al. Lack of Association Between Chemokine Receptor 5 (CCR5) delta32 Mutation and Pathogenesis of Asthma in Iranian Patients. South Med J. 2011;104(6):422-5. 